Proceedings

\title{
Recent Advances in High-Frequency Oscillations and Seizure Onset Detection Using Laplacian Electroencephalography via Tripolar Concentric Ring Electrodes ${ }^{\dagger}$
}

\author{
Oleksandr Makeyev ${ }^{1, *}$, Mark Musngi ${ }^{1}$, Frederick Lee ${ }^{1}$ and Michael Tamayo ${ }^{2}$ \\ 1 Department of Mathematics, Dine College, Tsaile, AZ 86556, USA; mmmusngi@dinecollege.edu (M.M.); \\ fjlee@dinecollege.edu (F.L.) \\ 2 Department of Electrical, Computer, and Biomedical Engineering, University of Rhode Island, Kingston, \\ RI 02881, USA; mickytamayo@my.uri.edu \\ * Correspondence: omakeyev@dinecollege.edu; Tel.: +1-92-8724-6960 \\ + Presented at the 4th International Electronic Conference on Sensors and Applications, 15-30 November \\ 2017; Available online: http://sciforum.net/conference/ecsa-4.
}

Published: 14 November 2017

\begin{abstract}
Laplacian electroencephalogram signal from novel and noninvasive tripolar concentric ring electrodes has been demonstrated to have superior performance compared to the electroencephalogram from conventional disc electrodes due to its unique capabilities which allow automatic attenuation of common movement and muscle artifacts in applications including braincomputer interface, seizure onset detection, and detection of high-frequency oscillations and seizure onset zones. This review paper covers the recent advances in the fields of high-frequency oscillations and seizure onset detection based on tripolar Laplacian electroencephalography in animal models and human data to improve the diagnostic yield of electroencephalography for epilepsy. Progression of methodologies utilized including integration of multiple sensors using exponentially embedded families and results obtained including a comparison to the results of others as well as to the performance of the same detector on simultaneously recorded electroencephalogram via conventional disc electrodes is discussed in detail. Specific advantages of using this particular sensor for these particular applications are highlighted. Promising directions for the future work and an overview of currently ongoing research are discussed along with the potential of combining the two detectors and using automatically detected high-frequency oscillations that have been shown to be indicative of early seizure development as auxiliary features for the seizure onset detection.
\end{abstract}

Keywords: tripolar concentric ring electrodes; Laplacian; high-frequency oscillations; seizure onset detection; epilepsy

\section{Introduction}

Epilepsy affects approximately 67 million people worldwide with up to $75 \%$ from developing countries [1]. Diagnosing epilepsy using electroencephalogram (EEG) is complicated due to its poor signal-to-noise ratio, high sensitivity to various forms of artifacts, and low spatial resolution. Detection of epileptic electrographic patterns can enhance diagnostic procedures during seizures and help to differentiate epileptic seizures from other conditions with seizure-like symptoms [2]. Automatic seizure detection also reduces the exhaustive manual review of EEG recordings. 
Therefore, improving the sensitivity and specificity of EEG as a predictor of seizure onset is of critical importance and would help reduce misdiagnosis and avoid over and under treatment.

Noninvasive tripolar concentric ring electrodes (TCREs) perform the second spatial derivative (surface Laplacian) to alleviate the blurring effects due to the skull and have very high common mode noise rejection providing automatic artifact attenuation, $-100 \mathrm{~dB}$ one radius from the electrode [3]. Compared to EEG with conventional disc electrodes Laplacian EEG via TCREs (tEEG) has been shown to have significantly better spatial selectivity (approximately 2.5 times higher), signal-to-noise ratio (approximately 3.7 times higher), and mutual information (approximately 12 times lower) [4]. Because of its ability to attenuate common movement and muscle artifacts tEEG found numerous applications in a wide range of areas including brain-computer interface $[5,6]$, seizure onset detection [7-11], detection of high-frequency oscillations (HFOs) and seizure onset zones [1,12], etc.

This review paper covers the recent advances in the fields of HFOs and seizure onset detection based on tEEG in animal model and human epilepsy patient data. There is growing evidence that increased HFOs within several frequency bands $>30 \mathrm{~Hz}$ may be indicative of early seizure development [13-15]. We have shown feasibility of recording HFOs with TCREs and their presence in tEEG prior to the onset of behavioral seizure manifestations in all our animal [12] and human epilepsy patient [1] seizure data. For seizure onset detection a progression of methodologies was proposed and validated including cumulative sum algorithm (CUSUM) [7] and generalized likelihood ratio test (GLRT) as well as disjunctive combination of the two [8] on pentylenetetrazole (PTZ) induced seizures in rats. Next an approach with three tEEG based features including median absolute deviation, approximate entropy, and maximum singular value serving as inputs to two classifiers including the support vector machines and adaptive boosting was validated on animal model tEEG and publicly available human epilepsy patient EEG data [9]. Unlike all the previous work where data from a single TCRE was used for seizure onset detection [7-9], in our recent studies data from multiple TCREs was integrated using the exponentially embedded family (EEF) approach that has been proposed for multi-sensor (or multi-channel) detection [16,17]. Applied to hypothesis testing, EEF has been shown to have superior performance compared to existing methods for cases where sensor outputs are not independent $[16,17]$. EEF based seizure onset detection has been validated on tEEG data both from the animal model [10] and human epilepsy patients [11]. The latter study included a comparison to the EEF performance on simultaneously recorded conventional EEG data [11] as well as a comparison to the EEG based results of others $[2,18,19]$.

\section{Advances in HFO Detection Using tEEG}

\subsection{Animal Model}

In [12] we demonstrated feasibility of recording HFOs up to $300 \mathrm{~Hz}$ in tEEG during PTZ-induced seizures in rats. We compared the power of electrographic activity at different stages of seizure development (baseline, ictal, and postictal) in a group of rats $(n=8)$ using grand average power spectral density estimates. The GLRT was used to assess statistical significance of differences in power of electrographic activity between the three stages. The results showed an increase in tEEG power at the ictal stage of seizure (assessed prior to the first myoclonic jerk to minimize the presence of seizure-induced movement artifacts) compared to the baseline stage that appears to be evenly distributed across the spectrum including the HFO bands of gamma activity $(30-80 \mathrm{~Hz})$, ripples (80$250 \mathrm{~Hz}$ ) and partial fast ripples $(250-300 \mathrm{~Hz})$. Moreover, the power of the ictal segments was significantly higher $(p=0.01)$ than the power of the baseline segments for all the animals $(n=8)$ in all evaluated frequency bands including the cumulative HFO frequency range $(30-300 \mathrm{~Hz})$.

The importance of this study stemmed from the fact that, unlike tEEG, EEG via conventional disc electrodes is limited in its ability to record HFOs from the scalp to ripples only [20]. 


\subsection{Human Data}

In [1] we demonstrated feasibility of recording HFOs with tEEG and their potential for early seizure onset detection and seizure onset zone detection on data from 5 epilepsy patients with clinical seizures. TCREs were placed directly behind the disc electrodes in the standard 10-20 system locations used in the hospital and the conventional disc EEG and tEEG were recorded concurrently. Looking at the data up to an hour prior to the onset of the seizures HFOs preceding seizures were present in tEEG data from all five patients whose seizures were recorded and absent in the corresponding EEG data. The average percentage of the total number of $t E E G$ channels recorded that contained HFOs was equal to 35.5\% (patient minimum: 5.3\%; maximum: $73.7 \%$ ). Out of all the tEEG channels that contained HFOs an average of 78.2\% (patient minimum: $42.9 \%$; maximum: $100 \%$ ) were also within the seizure onset or irritative zones determined independently by three epileptologists based on conventional EEG data and videos. While HFO activity may have occurred in the tEEG at other times during the recordings its bandwidth narrowed sharply and the power increased just prior to seizure onset.

This study confirmed that tEEG is capable of recording HFOs from scalp as well as attenuating myogenic activity and movement artifacts compared to conventional EEG and suggested the potential of recorded HFOs as markers of early seizure development. While the upper limit for detecting HFOs on the scalp is not established, in this study we have detected HFOs preceding seizures in gamma, ripple, and fast ripple bands at frequencies up to $425 \mathrm{~Hz}$ [1].

\section{Advances in Seizure Onset Detection Using tEEG}

\subsection{Animal Model}

Early CUSUM/GLRT based seizure onset detection methodologies proposed in [7,8] were based on detecting the changes in signal power since our previous results indicated a significant increase in $\mathrm{tEEG}$ power corresponding to seizure onset using population grand average power spectral density estimates [21] and frequency band analysis [7]. An average seizure onset detection accuracy of $76.14 \%$ was obtained for the test set $(n=13)$ with detection of electrographic seizure activity accomplished in advance of the early behavioral seizure activity in $76.92 \%$ of the rats [8].

In [10] EEF integrating data from three TCREs vas validated. While the overall accuracy obtained using EEG on a subset of the dataset from [8] was comparable to the one obtained using CUSUM/GLRT (81.7\% vs. 79.8\%), EEF outperformed CUSUM/GLRT with more than twice the sensitivity (69.4\% vs. $29.1 \%$ ) and comparable specificity (95.9\% vs. $98.3 \%$ ), higher percentage of rats with seizure onset detected prior to the first myoclonic jerk (100\% vs. $80 \%$ ), and faster response from PTZ injection to seizure onset detection (18.2 s vs. 26.6 s). Moreover, disjunctive combination (logical OR) of detections from EEF and CUSUM/GLRT did not show a significant improvement over detections from EEF alone suggesting near optimal performance of EEF for this dataset [10].

Improvement obtained using EEF in [10] is likely due to the fact that $t E E G$ via TCRE decreases mutual information, increasing the level of independency between electrodes compared to EEG via conventional disc electrodes [4]. Due to the significantly lowered mutual information multiple TCRE sensors collect more independent local data. Therefore, integration of multiple TCREs increases the total information possibly improving seizure onset detection. Further, with EEF the weights for each channel are estimated from the samples making it a robust method. In [10] we have shown that contribution of a channel is proportional to the amount of useful information it contains.

\subsection{Human Data}

In [11] EEF integrating data from 19 TCREs was validated on over $26.3 \mathrm{~h}$ of data collected from 7 human patients with epilepsy including five seizures. A non patient-specific model was used through $k$-fold cross-validation with each fold representing all the available data from a single patient to ensure direct comparison with results of others [2,18,19]. Sensitivity (correct detection rate), selectivity (false positive detections per hour, FPH), and detection delay were analyzed on EEG and 
tEEG data. For EEG data average sensitivity of $80 \%$, selectivity of $<1.56 \mathrm{FPH}$, and delay of $10 \mathrm{~s}$ were obtained. These results are comparable to results of others obtained on large scalp EEG datasets: in [18] sensitivity of $90.9 \%$, selectivity of $0.29 \mathrm{FPH}$, and delays of $10-44$ s were reported. In [2] sensitivity of $>96 \%$, selectivity of $<0.5 \mathrm{FPH}$, and average delay of $1.6 \mathrm{~s}$ were obtained. Finally, in [19] sensitivity of $76 \%$, selectivity of $0.34 \mathrm{FPH}$, and a median delay of $10 \mathrm{~s}$ were reported. More importantly, applying EEF to tEEG data we obtained average sensitivity of $100 \%$, selectivity of $<0.76 \mathrm{FPH}$ and delay of $31 \mathrm{~s}$.

This study demonstrated the following: first, the same EEF detector showed better performance on tEEG data compared to conventional EEG with higher sensitivity and less than half the FPH confirming the potential of using tEEG for seizure onset detection. Second, even on EEG data the EEF detector performed on par with the results reported by others while the results obtained with EEF on tEEG data suggested the possibility on improving on results of others with EEF applied to tEEG.

\section{Discussion}

Our recent seizure onset detector based on integration of multiple TCREs using EEF outperformed all the previously proposed detectors on animal model data [10]. Moreover, on data from human epilepsy patients this detector not only showed the results on par or better compared to the results of others $[2,18,19]$ but its performance on tEEG data was directly compared to performance on simultaneously recorded conventional EEG data once again confirming the advantage of TCREs over conventional disc electrodes [11]. Recording HFOs with scalp EEG is very difficult [20] but we recently showed on data from animal model [12] and human patients with epilepsy [1] that they can be recorded with tEEG.

The next fundamental direction of future work will be to assess the potential of using HFOs, that have been shown to be indicative of early seizure development [13-15], as auxiliary features for seizure onset detection. Adding a separate HFO detector or giving more weight to features in the HFO frequency range may not only improve the detection accuracy but decrease the detection delay allowing for a quicker seizure control. In [1] HFO detection was performed using visual inspection (i.e., manually) and only during up to an hour before the seizure onset on a dataset collected from a limited number of patients with epilepsy (five). In future, automatic HFO detection needs to be performed on complete tEEG recordings for patients with clinical seizures, patients with epileptiform activity but no seizures, and patients with neither epileptiform activity nor seizures. Such quantification of the presence of HFOs in between seizures (versus immediately preceding seizures) would allow conclusive assessment of the potential of using HFOs as an early predictor for seizure onset detection purposes. In order to create a separate automatic HFO detector several promising directions are currently pursued by our group. First, EEF may be used to detect HFOs if it is applied to power in individual or combined HFO bands (gamma activity, ripples, fast ripples) rather than to power across the spectrum as was done for seizure onset detection on tEEG data from the animal model [10] and human patients with epilepsy [11]. Second, the GLRT may be used to create an anomaly detector treating the $\mathrm{HFO}$ as a transient signal superimposed on a time-varying autoregressive background in order to localize events of interest. The localized event could be considered a wide-sense stationary process allowing a subsequent hypothesis test based on the frequency spectrum or detecting an oscillatory waveform characteristic of an HFO.

\section{Conclusions}

This paper reviews recent advances in HFO and seizure onset detection using tEEG via TCREs. Obtained results show feasibility of recording HFOs and detecting seizure onset early and reliably. Further improving tEEG based seizure onset detection using features derived from the automatically detected HFOs may be a key to development of better noninvasive diagnostic, therapeutic and alerting systems to alleviate the burden of epilepsy. 
Acknowledgments: The authors thank Walter G. Besio from the University of Rhode Island for the constructive discussions and helpful comments related to this research. This research was supported in part by the National Science Foundation (NSF) Division of Human Resource Development (HRD) Tribal Colleges and Universities Program (TCUP) award number 1622481 to Oleksandr Makeyev.

Conflicts of Interest: The authors declare no conflict of interest. The founding sponsors had no role in the design of the study; in the collection, analyses, or interpretation of data; in the writing of the manuscript, and in the decision to publish the results.

$\begin{array}{ll}\text { Abbreviations } \\ \text { EEG } & \text { Electroencephalography } \\ \text { TCREs } & \text { tripolar concentric ring electrodes } \\ \text { tEEG } & \text { Laplacian EEG via TCREs } \\ \text { HFOs } & \text { high-frequency oscillations } \\ \text { CUSUM } & \text { cumulative sum algorithm } \\ \text { GLRT } & \text { generalized likelihood ratio test } \\ \text { PTZ } & \text { pentylenetetrazole } \\ \text { EEF } & \text { exponentially embedded family } \\ \text { FPH } & \text { false positive detections per hour }\end{array}$

\section{References}

1. Besio, W.G.; Martinez-Juarez, I.E.; Makeyev, O.; Gaitanis, J.N.; Blum, A.S.; Fisher, R.S.; Medvedev, A.V. High-frequency oscillations recorded on the scalp of patients with epilepsy using tripolar concentric ring electrodes. IEEE J. Transl. Eng. Health Med. 2014, 2, 1-11.

2. Meier, R.; Dittrich, H.; Schulze-Bonhage, A.; Aertsen, A. Detecting epileptic seizures in long-term human EEG: A new approach to automatic online and real-time detection and classification of polymorphic seizure patterns. J. Clin. Neurophysiol. Off. Publ. Am. Electroencephalogr. Soc. 2008, 25, 119-131.

3. Besio, W.G.; Koka, K.; Aakula, R.; Dai, W. Tri-polar concentric ring electrode development for Laplacian electroencephalography. IEEE Trans. Biomed. Eng. 2006, 53, 926-933.

4. Koka, K.; Besio, W.G. Improvement of spatial selectivity and decrease of mutual information of tri-polar concentric ring electrodes. J. Neurosci. Methods 2007, 165, 216-222.

5. Besio, W.G.; Cao, H.; Zhou, P. Application of tripolar concentric electrodes and prefeature selection algorithm for brain-computer interface. IEEE Trans. Neural Syst. Rehabil. Eng. 2008, 16, 191-194.

6. Boudria, Y.; Feltane, A.; Besio, W. Significant improvement in one-dimensional cursor control using Laplacian electroencephalography over electroencephalography. J. Neural Eng. 2014, 11, 035014, doi:10.1088/1741-2560/11/3/035014.

7. Besio, W.G.; Liu, X.; Liu, Y.; Sun, Y.L.; Medvedev, A.V.; Koka, K. Algorithm for automatic detection of pentylenetetrazole-induced seizures in rats. In Proceedings of the 2011 Annual International Conference of the IEEE Engineering in Medicine and Biology Society, EMBC, Boston, MA, USA, 30 August-3 September 2011; Volume 2011, pp. 8283-8286.

8. Makeyev, O.; Liu, X.; Luna-Munguia, H.; Rogel-Salazar, G.; Mucio-Ramirez, S.; Liu, Y.; Sun, Y.L.; Kay, S.M.; Besio, W.G. Toward a noninvasive automatic seizure control system in rats with transcranial focal stimulations via tripolar concentric ring electrodes. IEEE Trans. Neural Syst. Rehabil. Eng. 2012, 20, 422-431.

9. Feltane, A.; Boudreaux-Bartels, G.F.; Besio, W.G. Automatic seizure detection in rats using laplacian EEG and verification with human seizure signals. Ann. Biomed. Eng. 2012, 41, 645-654.

10. Makeyev, O.; Ding, Q.; Kay, S.M.; Besio, W.G. Sensor integration of multiple tripolar concentric ring electrodes improves pentylenetetrazole-induced seizure onset detection in rats. In Proceedings of the 2012 Annual International Conference of the IEEE Engineering in Medicine and Biology Society, EMBC, San Diego, CA, USA, 28 August-1 September 2012; Volume 2012, pp. 5154-5157.

11. Makeyev, O.; Ding, Q.; Martínez-Juárez, I.E.; Gaitanis, J.; Kay, S.M.; Besio, W.G. Multiple sensor integration for seizure onset detection in human patients comparing conventional disc versus novel tripolar concentric ring electrodes. In Proceedings of the 2013 Annual International Conference of the IEEE Engineering in Medicine and Biology Society, EMBC, Osaka, Japan, 3-7 July 2013; Volume 2013, pp. 17-20. 
12. Makeyev, O.; Liu, X.; Wang, L.; Zhu, Z.; Taveras, A.; Troiano, D.; Medvedev, A.V.; Besio, W.G. Feasibility of recording high frequency oscillations with tripolar concentric ring electrodes during pentylenetetrazoleinduced seizures in rats. In Proceedings of the 2012 Annual International Conference of the IEEE Engineering in Medicine and Biology Society, EMBC, San Diego, CA, USA, 28 August-1 September 2012; Volume 2012, pp. 4599-4602.

13. Medvedev, A.; Mackenzie, L.; Hiscock, J.J.; Willoughby, J.O. Kainic acid induces distinct types of epileptiform discharge with differential involvement of hippocampus and neocortex. Brain Res. Bull. 2000, 52, 89-98.

14. Medvedev, A.V. Epileptiform spikes desynchronize and diminish fast (gamma) activity of the brain: An "anti-binding" mechanism? Brain Res. Bull. 2002, 58, 115-128.

15. Lévesque, M.; Langlois, J.M.P.; Lema, P.; Courtemanche, R.; Bilodeau, G.-A.; Carmant, L. Synchronized gamma oscillations $(30-50 \mathrm{~Hz})$ in the amygdalo-hippocampal network in relation with seizure propagation and severity. Neurobiol. Dis. 2009, 35, 209-218.

16. Kay, S.; Ding, Q.; Rangaswamy, M. Sensor integration for classification. In Proceedings of the 2010 Conference Record of the Forty Fourth Asilomar Conference on Signals, Systems and Computers, Pacific Grove, CA, USA, 7-10 November 2010; pp. 1658-1661.

17. Kay, S.; Ding, Q. Exponentially embedded families for multimodal sensor processing. In Proceedings of the 2010 IEEE International Conference on Acoustics, Speech and Signal Processing, Dallas, TX, USA, 1419 March 2010; pp. 3770-3773.

18. Hopfengärtner, R.; Kerling, F.; Bauer, V.; Stefan, H. An efficient, robust and fast method for the offline detection of epileptic seizures in long-term scalp EEG recordings. Clin. Neurophysiol. 2007, 118, 2332-2343.

19. Saab, M.E.; Gotman, J. A system to detect the onset of epileptic seizures in scalp EEG. Clin. Neurophysiol. 2005, 116, 427-442.

20. Zijlmans, M.; Worrell, G.A.; Dümpelmann, M.; Stieglitz, T.; Barborica, A.; Heers, M.; Ikeda, A.; Usui, N.; Le Van Quyen, M. How to record high-frequency oscillations in epilepsy: A practical guideline. Epilepsia 2017, 58, 1305-1315.

21. Makeyev, O.; Liu, X.; Koka, K.; Kay, S.M.; Besio, W.G. Transcranial focal stimulation via concentric ring electrodes reduced power of pentylenetetrazole-induced seizure activity in rat electroencephalogram. In Proceedings of the 2011 Annual International Conference of the IEEE Engineering in Medicine and Biology Society, EMBC, Boston, MA, USA, 30 August-3 September 2011; Volume 2011, pp. 7560-7563. 